\title{
Revista de la
}

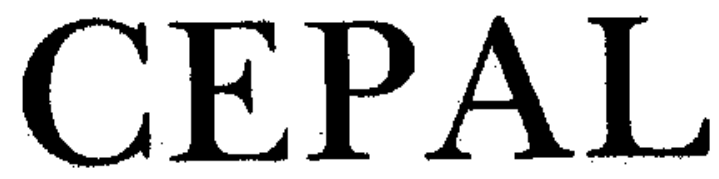

\author{
Secretario Ejecutivo \\ Norberto González \\ Secretario Ejecutivo Adjunto de \\ Desarrollo Económico y Social \\ Gert Rosenthal \\ Secretario Ejecutivo Adjunto de \\ Cooperación y Servicios de Apoyo \\ Robert T. Brown
}

\author{
Director de la Revista \\ Raúl Prebisch \\ Secretario Técnico \\ Adolfo Gurrieri \\ Secretaria Adjunta \\ Rosa Nielsen
}

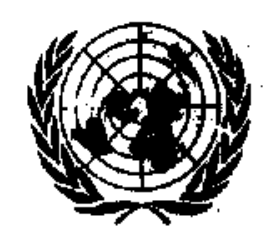




\section{Revista de la \\ C E P A L}

Santiago de Chile

Número 27

\section{SUMARIO}

Política exterior y negociación financiera internacional: la deuda externa y el Consenso de Cartagena. Jorge Eduardo Navarrete

7

Deuda externa: ¿por qué nuestros gobiernos no hacen lo obvio? Guillermo O'Donnell

Deuda externa y crisis: el ocaso de la gestión ortodoxa. Roberi Devlin

La deuda externa de los países latinoamericanos. Raúl Prebisch

América Latina y la integración: opciones frente a la crisis. Guillermo Maldonado Lince

Comercio y equilibrio entre los países de la Al.A1I. Jorge Torres Zorrilla y Eduardo Gana

Un ataque en dos frentes a la crisis de pagos de los países en desarrollo. Fabio R. Fiallo

Las fallas del mercado de capitales. Eduardo Sarmiento P.

La agricultura de América Latina: transformaciones, tendencias y lineamientos de estrategia. División Agricola Conjunta CEPAR/FAO

El papel de las empresas pequeñas y medianas en el mejoramiento de la estructura productiva de los paises en desarrollo. Carlo Secchi

25 años del Banco Interamericano de Desarrollo. Felipe Herrera

Algunas intervenciones realizadas en la Reunión de Expertos sobre Crisis y Desarrollo de América Latina y el Caribe (Santiago de Chile, 29 de abril al 3 de mayo de 1985) 


\section{REVISTA DE LA CEPAL N ${ }^{\circ} 27$}

\section{5 años} del Banco Interamericano
de Desarrollo

\author{
Felipe Herrera*
}

A partir de 1974, frente a las nuevas tendencias de la economía mundial, comenzó a perder progresivamente importancia relativa el financiamiento público internacional para los paises latimoamericanos. En efecto, el crecimiento de la liquidez monetaria internacional dio a la banca internacional una desconocicta gravilación en términos absolutos y relativos. Sin embargo, al persistir el proceso de recesion mundial, se vuelve evidente que nuestros paises debieran encontrar nuevamente la respuesta a sus necesidades en organismos como el Banco Interamericano de Desarrollo.

A juicio del autor, la experiencia de los últímos 25 anos en America Latina muestra que el financiamiento público internacional, desde cualqujera de sus perspectivas, consijtuye una forma de cooperación esencial para el progreso de la región.

La Revista de la cEPAL, ha querido destacar la impostancia del papel que desempeña el Banco Interamericano de Desarrollo con motivo de haber cumplido dicha institución un cuarto de siglo de actividades en el escenario regional e internacional. Valga la publicación de este artículo de su primer Presidente, señor Felipe Herrera, como homenaje a la institución hermana. rrolo.

\section{Algunos antecedentes de la creación del BID}

Pócos años después de terminar la segunda guerra mundial, los países de América Latina se vieron pospuestos en su empeño por obtener una cooperación financiera de mayor volumen, por la gran prioridad en escala internacional que se dio a la reconstrucción de Europa y al desarrollo económico de Africa y de algunas regiones asiáticas. También se criticaba en el continente en forma generalizada la rigidez de las políticas operativas de las fuentes públicas existentes. Ambos motivos de insatisfacción, junto con la antigua aspiración de contar con un sistema financiero regional propio, transformaron la creación de un banco regional en una bandera de lucha en los foros económicos interamericanos. Esta aspiración no fue compartida por los gobernantes norteamericanos de esos años, que no consideraban útil crear mecanismos nuevos, sino aprovechar más bien adecuadamente los recursos of recidos por el capital privado, por Eximbank y por el Banco Mundial.

La jnjciación del Plan Marshall, en 1947, alentó la esperanza de que los Estados Unidos, emulando el programa anunciado para Europa, pronto canalizaría importantes recursos para el desarrollo latinoamericano. Al no cumplirse esas expectativas, un sentimiento de frustración se puso de manifiesto en la Novena Conferencia Internacional Americana (Bogotá, 1948). En ella, los países de la región expresaron su desconformidad en cuanto al volumen de recursos que les asignaban los organismos públicos, tanto bilaterales como multilaterales. Para remediar esta situación se volvió a plantear la idea de crear un Banco Interamericano. En marzo del año siguiente, el Consejo Interamericano Económico y Social (CIEs) concluyó un estudio en el que analizaba los argumentos a favor y en contra de la creación de un organismo financiero regional, sin pronunciarse sobre el tema. Más adelante, en las sesiones extraordinarias celebradas en marzo y abril de 1950, ese organismo adoptó una resolución que declaraba: "en las circunstancias actuales, no parece factible o aconsejable la creación de instituciones de esta naturaleza".

En la Conferencia Económica Especializada 
(Quintandinha, noviembre de 1954) América Latina presentó, por primera vez, algunas ideas concretas acerca de las formas que debería revestir la cooperación económica interamericana. $\mathrm{A}$ pesar de que en la reunión prevaleció un ambiente de escepticismo frente a la viabilidad de la institución propuesta, por lo menos a corto plazo, se resolvió constituir una comisión de expertos, integrada por representantes de los bancos centrales de nueve países latinoamericanos, para que elaborara y presentara a la Organización de los Estados Americanos, en un plazo de seis meses, un proyecto de creación de una entidad financiera regional. El grupo de expertos se reunió en Santiago de Chile en febrero de 1955, y preparó un proyecto de estatutos que se remitió al cies, para que realizará las consultas del caso con todos los países miembros del sistema interamericano. Si bien este proyecto no tuvo consecuencias inmediatas, $y$ la idea de crear un banco interamericano no volvió a debatirse hasta dos años después, la reunión de Santiago preparó un marco adecuado para que esta antigua aspiración latinoamericana fuera objeto de reflexión sistemática.

En la Conferencia Económica de la OEA (Buenos Aires, agosto y septiembre de 1957) se inició un proceso de cambio en las relaciones interamericanas que culminó con la Operación Panamericana, propuesta por el Presidente de Brasil, doctor Juscelino Kubitschek, y respaldada por todos los paises miembros del sistema. La Operación Panamericana constituyó un antecedente decisivo para la creación del BtD, no sólo porque incluia un mecanismo de esta especie entre las medidas cuya adopción proponía, sino también porque influyó para que Estados Unidos decidiese reexaminar su política de cooperación al desarrollo económico y social de América Latina.

Cabe recordar que en ese entonces el Presidente Eisenhower, al proponer la creación de una Agencia de Desarrollo Regional para el Medio Oriente, con la participación financiera de su pars, habia aceptado la posibilidad de incorporar instrumentos regionales de desarrollo en los sistemas globales existentes. El 12 de agosto de 1958, en una sesión especial del cirs, el subsecretario de Estado anunció que los Estados Unidos estaban preparados para participar en una orga- nización financiera regional como la propuesta para América Latina, lo que coincidió con la convocatoria a una reunión de Ministros de Relaciones Exteriores que debía celebrarse al mes siguiente a fin de analizar los planteamientos en que se fundaba la Operación Panamericana. En esa oportunidad los países del sistema interamericano apoyaron la operación propuesta por el Presidente Kubitschek y se constituyó un grupo de expertos encargados de negociar el convenio constitutivo del nuevo organismo financiero regional. Con esta decisión se abría la última etapa en la creación del BID.

La creación del Banco hizo realidad la antigua aspiración de los países latinoamericanos de contar con un instrumento financiero de carácter regional en cuya gestión ellos tuvieran participación efectiva. Su largo proceso de gestación es fruto de los esfuerzos realizados por conciliar, en la institución proyectada, sus caracteristicas latinoamericanas y su plena incorporación en el sistema interamericano. Regionalismo y multilateralidad definen, por lo tanto, el carácter y la esencia del nuevo organismo. Las características regionales originales del BID fueron ampliadas a partir de 1976, transformándose la entidad en una organización internacional con el ingreso de Alemania occidental, Austria, Bélgica, Dinamarca, España, Finlandia, Francia, Italia, Israel, Japón, los Países Bajos, Portugal, Reino Unido, Suecia, Suiza y Yugoslavia.

En lo personal, especialmente en mi calidad de Gerente General del Banco Central de Chile y luego, como Director Ejecutivo del Fm, en representación del Cono Sur, me correspondió entre 1954 y 1959 la honrosa tarea, en nombre de mi pais, primero, y luego, de otras naciones hermanas, de participar en la promoción de la iniciativa. Fui miembro de la comisión redactora y organizadora del BID, y luego, en la Primera Asamblea de Gobernadores del в id (San Salvador, enero de 1960) fui elegido Presidente de la institución, siendo reelegido en 1964 y 1968.

Al agradecer mi nombramiento en San Salvador, me permití expresar, en relación con el desafio que teníamos por delante, lo siguiente: "La dimensión y calidad del Banco estarán determinadas, por una parte, por los factores humanos y de organización y, por otra, por el volumen de los recursos disponibles y de las modalidades 
con arreglo a las cuales éstos se aprovecharán. El éxito del Banco dependerá fundamentalmente de los hombres que lo manejen. Tenemos que esclarecer el verdadero alcance del Banco Interamericano y destacar que sólo comienzos serenos, fundamentados y con claridad de objetivos, permitirán a la institución crecer e ir asumiendo mayores responsabilidades. Necesitamos que quienes tanto esperan de nosotros, nos otorguen tiempo y buena voluntad en este período de formación y crecimiento. Con viva preocupación buscaremos los medios más adecuados para vincular el Banco con la conciencia colectiva de nuestra gente. Se juzgó necesario que la sede de la institución estuviera en Estados Unidos por convincentes argumentos técnicos y prácticos. Esta circunstancia, sin embargo, nos obliga a estar más alertas que nunca, recordando que el.
Banco existe para el servicio de América Latina; la institución y su personal deben sentirse identificados con la vigorosa y apasionante realidad latinoamericana. Debemos crear una institución sólidad y eficiente que gane la confianza y atraiga el interés de los mercados de capitales; pero también debemos estar dispuestos, con la misma decisión y con el mismo convencimiento, a que el Banco viva y se desarrolle arraigado a nuestras colectividades. Las variadas motivaciones que influirán en las actividades del Banco Interamericano de Desarrollo y las modalidades propias del escenario en que tendrá que actuar indican lo compleja que será nuestra institución. Es ella una organización bancaria, especializada en el financiamiento regional de obras de desarrollo económico. Sin embargo, siendo un Banco, es 'algo más que un banco" ".

\section{II \\ La tarea del BID}

En el largo proceso de gestación y negociación a que se ha hecho referencia, se creo un Banco con objetivos y características precisos, establecidos en su propio Convenio Constitutivo; sin embargo, fue la actividad propia del bịo la que delimitó sus contornos y definió su imagen y naturaleza.

Cabe señalar, en primer término, su capacidad de movilización de recursos, no sólo entre sus socios fundadores, sino también en otros paises desarrollados. En efecto, un ente regional ofrece inmejorables perspectivas de coadyuvar, con los paises beneficiarios de la ayuda, a generar contrapartida locales adecuadas. En este sentido fue muy bien lograda la experiencia del BID ya que por cada unidad de moneda extranjera prestada se comprometian fondos locales equivalentes por lo menos al doble.

Como es evidente, en este proceso de estimular la inversión local en función del aporte externo se tiende a perfeccionar todo el mecanismo de ahorro e inversión en los países en vías de desarrollo, tanto en el ámbito público como privado.

Por otro lado, es evidente que la institución se ha desempeñado eficazmente en campos de acción no cubiertos antes por otras entidades. Especialmente, debemos mencionar:

a) La promoción del desarrollo equilibrado del continente, a cuyo objeto se han adoptado políticas tendientes a dar atención prioritaria a los países de menor desarrollo relativo y a las zonas rezagadas dentro de una misma nación. También en EI Salvador, me permití plantear mi criterio al respecto en los siguientes términos: "Es un hecho que en nuestro continente, formado por naciones y regiones en proceso de desa. rrollo, existen áreas que, por complejas razones, están más atrasadas que otras. En nuestra concepción de la unidad económica de América Latina, esta realidad no nos puede dejar indiferentes. Tal como en el marco de la economía nacional el retraso de una parte del territorio puede crear serios problemas a toda la colectividad, así también en el continente, zonas o naciones de notorio subdesarrollo pueden significar peligrosos desajustes y tensiones para el futuro económico, politico y social de nuestra comunidad de naciones. Debemos, pues, y de acuerdo con los recursos disponibles y las modalidades de nues- 
tros estatutos, dar preferencia a la solución de estos problemas. No olvidemos que no hemos creado una institución para naciones aisladas, sino para un continente en su vigoroso conjunto".

b) Un alto porcentaje de la cartera del Banco ha estado constituida por préstamos dedicados al fortalecimiento de la infraestructura física regional, ya que sus deficiencias limitan severamente el rendimiento de cualquier inversión reproductiva. Sin una red articulada de transportes y telecomunicaciones y una explotación más intensa de los recursos energeticos, se acentuaban las tendencias deformantes de un desarrollo centralizado en pocos polos desconectados entre sí, lo que dificultaba la conquista de la América interior y los procesos de integración regional y nacional.

c) El financiamiento internacional del sector agropecuario habia sido desatendido hasta la creación deì Banco, cuya acción en este campo ha tenido por lo tanto un valor pionero y de promoción. Las operaciones aprobadas para proyectos de riego, diversificación agrícola, desarrollo ganadero, zoonosis y comercialización, hacen del biD el principal proveedor de capital externo para el desarrollo agrícola de América Latina.

d) $\mathrm{El}$ apoyo decidido al sector industrial se manifestó en el financiamiento masivo en favor de empresas nacionales, a través de préstamos globales concedidos a corporaciones de fomento y bancos industriales, así como con operaciones directas y financiamiento de las exportaciones intrarregionales de bienes de capital. La importancia de este último programa no se puede medir tan sólo por las cifras de intercambio conseguido, sino más bien por su efecto promotor. También ha constituido un estímulo para la industria latinomericana la adquisición, con préstamos del Banco, de bienes y servicios en paises latinoamericanos y muchos de ellos han sido proveedores para proyectos financiados por el Banco. En el discurso mencionado, al hacerme cargo de la primera presidencia del Banco, destaqué que: "Doy grande importancia, en la práctica de las operaciones del Banco, a las posibilidades que éste tiene de actuar a través de organismos nacionales destinados a ayudar al financiamiento del desarrollo económico, sean corporaciones de fomento, bancos de desarrollo, bancos agricolas 0 industriales 0 entidades financieras similares. La experiencia de estas organizaciones y su conocimiento de la economía y de las necesidades de sus respectivos países, pueden transformarlas en hábiles canalizadoras de parte de los recursos del Banco. Existen importantes áreas atendidas generalmente por empresas privadas, medianas y pequeñas, en que podemos concurrir con nuestros fondos $y$ multiplicar sus proyecciones. En este sentido es en el campo agropecuario y en el de la industrialización donde nuestra acción tal vez pueda ser de necesidad y resultado máximos. Para estos fines debemos buscar también el apoyo de fuentes privadas de financiamiento local, especialmente a través de entidades de capitalización y bolsas de valores. Los conceptos anteriores nos permiten prever que la cooperación del Banco Interamericano de Desarrollo con los organismos de fomento, con la banca privada $y$ central y con los mercados financieros de cada pais, será naturalmente muy estrecha".

e) El Banco ha prestado atención preferente al desarrollo urbano para hacer frente al intenso proceso de concentración en las ciudades latinoamericanas, derivado de la modernización del agro y del avance de la industrialización. El Banco ha atribuido gran importancia a la cooperación financiera, técnica e institucional para promover la creación y el mejoramiento de la infraestructura en los países miembros.

f) Como la capacidad tecnológica constituye uno de los factores más dinámicos para el desarrollo, se há prestado apoyo financiero a la educación y al adiestramiento superior. El Banco se ha asociado al esfuerzo de los paises latinoamericanos por aumentar la capacidad y mejorar la calidad y la eficiencia de su educación universitaria y técnica. Las operaciones del sID se han dirigido principalmente a reforzar las instituciones académicas más eficaces para la formación de recursos humanos destinados a las actividades productivas, y a introducir a a consolidar refor* mas de fondo en la organización de las instituciones educativas, así como en la metodología de la enseñanza, de lá investigación científica y de la tecnología.

g) No puede desestimarse la importancia de un banco regional en la creación de una infraestructura institucional para el desarrollo. La acción prestamista sirve de estímulo a todo el pro- 
ceso de planificación, que tiene una interesante evolución en el decenio de 1960, asi como a la creación y al mejoramiento de organismos de fomento de diversa índole. Así también, un organismo regional sirve como verdadera universi- dad para el desarrollo, no sólo por el intercambio de experiencia entre sus funcionarios, sino por la proyección que éstos tienen cuando vuelvan a ocupar posiciones claves en sus respectivos países.

\section{III}

\section{Proyecciones innovadoras del BID}

En toda esta labor se ha tratado de actuar con criterio pragmático, armonizando la inversión económica con la social, los financiamientos 'duros' de proyectos altamente reproductivos con los financiamientos 'blandos'. Se ha procurado atenuar el desequilibrio entre los países con distintos niveles de desarrollo, y aun entre zonas de desarrollo desigual dentro de un mismo pais. Se ha intentado superar la barrera entre el sector público y el privado, teniendo como norma para ello la decisión de los propios gobiernos: se ha apoyado al Estado y a la empresa pública, si en opinión del gobierno eran éstos los agentes principales del desarrollo nacional; o bien a la empresa privada, si éste decidía dar esa orientación a los recursos internacionales.

El criterio rector ha sido la eficiencia y el mejor aprovechamiento de los recursos. $Y$ junto a esta interpretación fiel de los objetivos y las metas trazadas soberanamente por los propios países, se ha procurado cultivar la capacidad de innovación, requisito ineludible para mantenerse en vigencia en un escenario caracterizado por la velocidad del cambio. La continua búsqueda de nuevos campos de financiamiento, desde la educación al turismo, desde la salubridad hasta el financiamiento de exportaciones o la explotación de los recursos del mar, a pesar del riesgo que estas innovaciones implican, tuvo como consecuencia que otros organismos internacionales siguieran los pasos del BIv. En el citado discurso de El Salvador, me permiti plantear lo siguiente: "Estas ideas nos Ilevan a concluir que, en la práctica, el Banco superará las distinciones artificiales que teóricamente se quieren acentuar entre sector público y sector privado. El grado de importancia de uno u otro, en la organización económica de un país depende de su particular estructura institucional. No corresponde al Banco interferir en este sentido. La coexistencia de empresas públicas y privadas es un hecho dado en nuestras colectividades: al Banco en su política de préstamos sólo debe interesarle el grado de eficiencia con que se manejen esas actividades y la utilidad que para ellas, y para la economía nacional en conjunto, pueda representar la utilización de sus fondos".

El crecimiento de sus préstamos 'blandos' permitió al Banco lograr rápidamente un equilibrio adecuado entre sus operaciones de contenido social y el financiamiento de proyectos economicos que constituía su actividad fundamental. Esta original dimensión del Banco como organismo de financiamiento del desarrollo social latinoamericano, se encuentra en embrión en las disposiciones que regulan su Fondo para Operaciones Especiales, establecido en su Convenio Constitutivo. Al discutirse la idea del Banco Interamericano de Desarrollo existía ya la noción de que el desenvolvimiento equilibrado de los países de la región haria necesario atender proyectos menos convencionales o de menor productividad directa, que por su naturaleza misma requerían financiamiento en condiciones más flexibles. Sin embargo, la labor desplegada por el Banco en los sectores sociales $y$, con ello, las actividades del Fondo para Operaciones Especiales, se incrementaron notablemente a partir del Acta de Bogotá, suscrita al finalizar el tercer periodo de sesiones de la Comisión Especial del Consejo Interamericano Económico y Social en septiembre de 1960, es decir, meses antes que se pusiera en vigencia el Programa de la Alianza para el Progreso del Presidente J.F. Kennedy.

El Acta de Bogotá significó para el Banco y para toda América Latina no sólo la adopción de 
modalidades operativas hasta entonces desconocidas, sino también la aceptación de nuevas concepciones doctrinarias. En ella se reconoce que el impacto de los programas de desarrollo econó mico en el bienestar de la población tiende a manifestarse a largo plazo, y, por consiguiente, propone un conjunto de medidas de mejoramiento social, sobre todo en materia de desarrollo rural, vivienda, educación y salud. Al suscribirse el Acta, Estados Unidos anunció que estaba dispuesto a poner 500 millones de dólares al servicio de sus objetivos. El Congreso de ese país asignó esos recursos de la siguiente forma: 6 millones de dólares a la Organización de los Estados Americanos para colaboración con los países miembros en la preparación de sus planes de desarrollo; 100 millones de dólares a la International Cooperative Administration (ICA), para donaciones o asistencia bilateral en educación, salud pública y asistencia técnica; y 394 millones de dólares a la formación del Fondo Fiduciario de Progreso Social, que sería administrado por el BID, para apoyar los esfuerzos de los países latinoamericanos que estuvieran dispuestos "a iniciar o ampliar mejoras institucionales efectivas $y$ a adoptar medidas para utilizar eficazmente sus propios recursos, con miras a alcanzar un mayor progreso social y un crecimiento económico más equilibrado". El 19 de junio de 1961, cuando el
Bro acababa de iniciar sus actividades, se firmó el Contrato de Fideicomiso entre el Gobierno de los Estados Unidos y el Banco que autorizaba a este último a efectuar préstamos con cargo a ese Fondo en los campos de la colonización y el mejor uso de la tierra, la vivienda para sectores de bajos ingresos, el saneamiento ambiental, y la educación y el entrenamiento superior. Se autorizó también al Banco a suministrar asistencia técnica en esos sectores. De este modo el nuevo Fondo otorgó carta de ciudadanía al financiamiento social entre las políticas operativas de la institución.

Al principio habia cierta preocupación por la posibilidad de que el Fondo Fiduciario pudiera deformar el concepto y las actividades del Banco, el apartarlo de proyectos preponderantemente económicos. Cabe recordar que en aquella época, cuando los países miembros habían pagado apenas la primera cuota de sus contribuciones a los recursos ordinarios y especiales del Banco, el Fondo Fiduciario de Progreso Social representaba un incremento considerable de recursos. Hoy se puede apreciar que no hubo tal distorsión y que, si bien el Banco amplió en gran medida su participación en el financiamiento de la inversión social en América Latina, lo hizo sin menoscabo de la atención que prestaba a los sectores económicos.

\section{IV}

\section{El banco de la integración}

Orras de la funciones del Banco, implicita en su Convenio Constitutivo y que se fue desarrollando en forma paulatina con el correr de los años, radica en su papel como promotor de la integración económica latinoamericana. Aunque de alguna manera la expresión "desarrollo colectivo", contenida en la Carta básica, encerraba ya el concepto de la integración regional, las disposiciones del Convenio que definen las funciones del Banco la refieren, fundamentalmente, al financiamiento de proyectos y actividades de carácter nacional. Sin embargo, confiriendo a la acción del вID una proyección más amplia, una de esas disposiciones le asigna la misión de "cooperar con los paises miembros a orientar su politica de desarrollo hacia una mejor utilización de sus recursos, en forma compatible con los objetivos de una mayor complementación de sus economías y de la promoción del crecimiento ordenado de su comercio exterior". Aunque esos conceptos no contienen todo lo que ahora se entiende por integración económica, había en ellos un mandato que la evolución ulterior de América Latina y del propio Banco Interamericano contribuyeron a esclarecer.

Para ello, el Banco tuvo que participar en el 
rico proceso intelectual de esclarecimiento del significado y de los alcances de la integración económica regional, concepto que sólo viene a consolidarse al despuntar el decenio de 1960. En la actualidad, es frecuente oir hablar en Estados Unidos de los golden sixties; nos parece que esta definición es también muy apropiada para el proceso de reencuentro latinoamericano y de maduración de perspectivas para la convergencia histórica entre nuestros países en esa década.

A medida que crecía la influencia intelectual del Banco en el proceso de integración latinoamericana, aumentaba la inquietud por dotar a estas ideas de una base operativa. En la cuarta Reunión de la Asamblea de Gobernadores (Caracas, 1963), se puso en marcha un mecanismo para el financiamiento de las exportaciones intrarregionales de bienes de capital, cuyo objetivo era colocarlas en condiciones de competencia frente a las provenientes de otras regiones. El programa funcionaba originalmente a base de líneas de crédito a organismos financieros nacionales, y para comenzar contó con una asignación de 30 millones de dólares provenientes de los recursos ordinarios del Banco. El éxito del programa puede apreciarse por el volumen de comercio que ha fomentado; por la naturaleza de. los bienes intercambiados, que constituyen una innovación en las corrientes comerciales tradicionales de la región; y por las mejoras institucionales que de él derivan. La utilización de una parte de los recursos venezolanos puestos en 1975 a disposición del Banco para ampliar el financiamiento de exportaciones regionales y la creación en 1978 del Banco Latinoamericano de Exportaciones (BLADEX) con sede en Panamá constituyen, a mi entender, interesantes proyecciones de la experiencia de este programa.

La consagración definitiva del ва como Banco de la Integración data, tal vez, de la Sexta Reunión de la Asamblea de Gobernadores (Asunción, 1965). En esa oportunidad se expresó el apoyo unánime al denominado "Documento de los 4", es decir, al informe para acelerar la creación del mercado común latinoamericano que, a sugerencia del Presidente Frei, habían preparado pocas semanas antes para todos los Jefes de Estado de] Hemisferio, Raúl Prebish (ILPES), Carlos Sanz de Santa María (ciap), José A. Mayobre (CEPAL), y el autor de este artículo, en su calidad de Presidente del BID. En la parte final del documento reiteramos nuestra fe en el futuro de una América Latina integrada en los términos siguientes: "Hay que aprender a trabajar en comunidad; debemos formar la comunidad de pueblos latinoamericanos. Todavía no hemos sido capaces de afrontar esta gran tarea en medida adecuada, porque no hemos logrado desembarazarnos completamente del molde en que comenzo a operarse nuestro desarrollo en el siglo xix. Separados entre sí, sin activas relaciones que los uniera vitalmente, cada uno de nuestros países convergía entonces en forma aislada hacia los grandes centros mundiales de la economía, la política y la cultura. Viviamos del reflejo que esos centros irradiaban, y ello tuvo consecuencias de profunda huella en el pasado de Latinoamérica y sigue teniéndola en los tiempos presentes. Subsisten todavia importantes elementos de ese esquema; y se impone deshacernos de ellos frente a la evolución del mundo y las tensiones crecientes de nuestro desarrollo. Es imperioso combinar nuestras fuerzas, para superar esos y otros obstáculos que se oponen al desarrollo latinoamericano, y concretarlas en la realización de grandes objetivos comunes. No se trata solamente de responder a las exigencias de la técnica, ni de que tengamos que trabajar en comunidad para lograr un gran espacio económico y dilatar el horizonte cultural, científico y tecnológico. Necesitamos también hacerlo para alcanzar una mayor gravitación política en el plano internacional".

La participación del Banco Interamericano en el proceso de integración se tornó más eficaz por efecto de dos iniciativas: la creación del Instituto para la Integración de América Latina (INTAL) y el ulterior establecimiento del Fondo de Preinversión para la Integración de América Latina. La idea de establecer un instituto de esta naturaleza había sido ya propuesta en la Reunión de alalci celebrada en la Ciudad de México en 1963. Entonces se planteó la necesidad de crear un mecanismo coordinador de las diversas instituciones de integración existentes en América Latina. Como pasara el tiempo sin que nada sucediera en ese sentido, el Banco decidió establecer una institución propia llamada a cumplir funciones de adiestramiento, investigación, difusión y asesoramiento en relación con el proceso de integración económica regional. 
En 1966 se estableció el Fondo de Preinversión para la Integración de América Latina, en cumplimiento del mandato específico de la Asamblea de Gobernadores. El Fondo se constituyó inicialmente con 15 millones de dólares, tomados de los recursos para operaciones especíales, suma a la cual el Gobierno de Estados Unidos agregó otros 3.5 millones de dólares provenientes del Fondo Fiduciario de Progreso Social. Entre los campos de acción del Fondo se contaban, ante todo, la ejecución de proyectos de infraestructura de alcance integracionista, sobre todo en materia de transporte y comunicaciones, así como la organización de esos servicios, a escala regional, para el aprovechamiento de espacios económicos que comprendían territorios pertenecientes a dos o más países; el establecimiento o la expansión de industrias básicas que operaban para mercados multinacionales o contuvieran insumos procedentes de varios países, etc. El Fondo fue de gran utilidad para acelerar la comple- mentación de las economías de la región, ya que facilitó la identificación de nuevas posibilidades multinacionales de inversion. Todo lo anterior tuvo una nueva ratificación en la declaración de Punta del Este de 1967, en que los Jefes de Estado del Sistema Interamericano reconocieron la labor prioritaria que le correspondía al BID en el financiamiento de proyectos de infraestructura multinacional con miras a seguir acelerando el proceso de alcanzar la unidad económica latinoamericana. Es un desafio para el BID transformarse efectivamente en un banco de la integración. De los préstamos del Banco, en su conjunto no más de un $10 \%$ de los fondos comprometidos se dedican a proyectos multinacionales; de ahí que reafirmamos nuestra convicción de que el nm debe ser un instrumento promotor de la convergencia financiera latinoamericana, proyectada en iniciativas conjuntas diversas de dos o más parses.

\section{V \\ Comentarios finales}

Como es obvio el medio financiero-político que rodea al Banco es diverso en 1985 de lo que fue en sus etapas iniciales. Recordemos que a principios del decenio de 1960 el interamericanismo económico correspondía a un nuevo concepto de la responsabilidad de los Estados Unidos frente a América Latina; su mejor expresión fue la Alianza para el Progreso. A partir de 1968, Nixon y Kissinger cambian las reglas del juego con una política general de abandono progresivo de la transferencia de recursos públicos de los países industrializados hacia el Tercer Mundo.

En los primeros años del decenio de 1960, el proceso latinoamericano de integración y regionalización arrancó poderosamente con la ALALG: y el mercado común centroamericano. En años recientes, esa tendencia tömó otro rumbo, dándose mayor importancia a la convergencia sectorial. El Sistema Económico Latinoamericano (SELA) expresa esta realidad. El BIo ha logrado una presencia permanente en estos sucesos y ha financiado importantes proyectos multinacionales de infraestructura.

En mayo de 1985 el intal convocó a un seminario destinado a analizar el problema del financiamiento de los organismos subregionales. Contó con la activa participación del Fondo para el Desarrollo de la Cuenca del Río de la Plata (FonPlata), del Banco Centroamericano de Integración Económica y de la Corporación Andina de Fomento (CAr). En esa reunión se destacó el apoyo que había dado el BiD a todos los sistemas de financiamiento subregional, asi como las dificultades actuales de estas instituciones por las limitaciones derivadas de la situación de endeudamiento global de América Latina.

El Banco nació en un periodo en que América Latina se caracterizaba por sus vacilantes tasas de desarrollo, relación negativa de intercambio y bajas reservas monetarias. En el decenio de 1970 la región alcanzó índices de crecimiento de $6 \%$ a $8 \%$; aumentaron y se diversificaron sus exporta- 
ciones; $y$ sus reservas monetarias subieron de 3000 a más de 30000 millones de dólares. En ese contex to el volumen de crédito que podían proporcionar los organismos internacionales para muchos países tenía menor trascendencia, ya que habían nuevas posibilidades de inversión interna y externa, producto de las cambiantes condiciones del comercio y del financiamiento internacional. Desgraciadamente, en el curso de ese decenio, por nuevas condiciones económicas y políticas, en vez de la maduración hacia el regionalismo latinoamericano, se acentuaron mal entendidos principios de libertad económica y de sustitución de una generalizada tradición democrática. En los últimos años, sin embargo, se han visto renacer las convicciones integracionistas y democráticas en América Latina, lo que, paradójicamente, en muchos casos se ha debido a las nuevas políticas que se practicaron y que llevaron al conjunto de la región a un excesivo endeudamiento financiero externo.

En la composición interna del conglomerado latinoamericano se produjeron también cambios importantes que influyeron en la acción del BiD. A principios del decenio de 1960 Brasil era considerado aún, para los financistas internacionales, como un riesgo (le correspondió al BID volver a abrir para este país la llave del crédito público internacional). Aunque Venezuela luchaba por consolidar su democracia y estimular su desarrollo económico interno, estaba muy lejos de su espectacular bonanza. En Sudamérica, las condiciones prevalecientes en Bolivia, Paraguay y Ecuador reflejaban un agudo subdesarrollo que, en estos últimos tres lustros, tiende a superarse. Los nuevos paises del Caribe, independizados del Reino Unido y los Paises Bajos o en proceso de descolonización, no formaban parte del sistema regional, siendo casi inexistentes sus vinculaciones con la mayoría de las naciones latinoamericanas. En cambio, entre 1967 y 1969 se incorporaron al sis Trinidad y Tabago, Barbados, $y$ Jamaica, y más recientemente se han asociado Guyana, Bahamas y Suriname.

$\mathrm{Al}$ establecerse el bid, el único país industrializado que había comprometido grandes recursos con la institución era Estados Unidos. Desde 1962, el Banco comenzó a conseguir la colaboración financiera de todos los países exportadores de capital. En 1972, Canadá se convirtió en miembro de la institución y a partir de 1976, 17 naciones desarrolladas extrarregionales se transformaban en accionistas de la entidad, con lo que dejó de ser un ente estrictamente hemisférico para adquirir un carácter cada vez más cosmopolita.

Una de la pruebas de fuego para el Banco fue su acceso a los mercados internacionales de capital, lo que consiguió a lo largo de estos años en óptimas condiciones con el respaldo técnico de una cartera de primera categoría.

La actividad operativa del Banco fue considerada por muchos, en sus inicios, como heterodoxa, al superar pragmáticamente la antinomia entre préstamos convencionales y blandos; entre sector público y sector privado; entre agricultura $\mathrm{e}$ industria; entre inversión económica e inversión social; entre inversión y preinversión; entre financiamiento y asistencia técnica. El вир fue caracterizándose así por ser el banco central de los bancos de fomento de América Latina, el banco de la integración, un verdadero banco de ideas.

A veces se le ha criticado como banco manejado por sus deudores, es decir con espíritu y mayoría latinoamericanos, atribuyéndose a la institución una supuesta politización. Me correspondió contestar esos comentarios, al terminar mis funciones, en marzo de 1971, expresando: "Si este Banco no hubiera tenido la inquietud y la angustia de un desarrollo acelerado en América Latina con un sentido de integración y en beneficio de las mayorías, todo ello motivación política, no sería lo que es. Y como en la década de los setenta es muy probable que el cuadro pluralista de América Latina se amplie y complique, creo que necesitaremos aguzar nuestra buena voluntad y nuestra inteligencia para poder encontrar fórmulas de coincidencia para conjugar las fuerzas centrífugas crecientes; y la única forma de lograr tal conciliación, es recurriendo a criterios políticos superiores".

Al asumir la Presidencia del Banco, en 1960, me permití detinir nuestra responsabilidad frente a un Banco que al mismo tiempo "era algo más que un Banco". Creo que tratê de ser consecuente con esta inspiración durante toda una década, según lo expresó al terminar mi mandato, en marzo de 1971 , y al hacerle transferencia de estas 
funciones al nuevo Presidente de la institución, mi distinguido y eficiente amigo Lic. Antonio Ortiz Mena, en los términos siguientes: "Quizás hoy percibamos con más claridad todo lo que intuimos hace once años cuando dijimos que el BID era algo más que un Banco: una gran organismo público regional, sólidamente establecido por su prestigio técnico y su capacidad financiera, en el cual participan los paises industriales pero que no por ello deja de ser profundamente latinoamericano y cuyo objetivo último no es sólo la modernización de las estructuras productivas, no es sólo el simple crecimiento económico, sino la elevación de los niveles de bienestar de las grandes masas latinoamericanas. Es algo más que un Banco por la orientación creciente de su esfuerzo institucional hacia la promoción del desarrollo integrado de nuestros pueblos y porque se ha convertido en caja de resonancia y en vocero de los intereses de América Latina. En esta contribución que el Banco puede y debe hacer para que los pafses y la región en su conjunto encuentren su propia vía de desarrollo con independencia, como incitación a la audacia creadora, valga recordar una frase de ese inolvidable servidor público, Secretario General de las Naciones Unidas, Dag Hammarskjold: "No escudrines el suelo antes de dar tu próximo paso: sólo aquel que mantiene sus ojos fijos en el lejano horizonte encontrará su verdadero camino". 